\title{
Electrophoretic Karyotypes of Authentic Strains of the Sensu Stricto Group of the Genus Saccharomyces $\dagger$
}

\author{
GIANLUIGI CARDINALI AND ALESSANDRO MARTINI* \\ Dipartimento di Biologia Vegetale, Sezione Microbiologia Applicata, Industrial Yeasts \\ Collection DBVPG, University of Perugia I-06121 Perugia, Italy
}

\begin{abstract}
A comparative electrophoretic karyotyping study was performed with several certified authentic strains of the four species that could be distinguished by nuclear DNA (nDNA)-nDNA reassociation data within the sensu stricto group of the genus Saccharomyces. A multivariate analysis of the polymorphisms observed in pulsed-field gel electrophoretic profiles (numbers and molecular weights of separated units) revealed that the strains could be separated into four clusters that corresponded to the taxa that were distinguished on the basis of nDNA comparisons. Discrepancies between nDNA reassociation data and membership in the corresponding clusters were observed only with two strains of Saccharomyces paradoxus. Blind tests carried out with additional industrial strains confirmed the general validity of the statistical model created for comparison of karyotypes within the species included in Saccharomyces sensu stricto.
\end{abstract}

The species assigned by van der Walt (30) to the sensu stricto group of the genus Saccharomyces include the microorganisms that have been exploited industrially and scientifically by humans for the longest time. Innumerable ecological and technological investigations have redundantly demonstrated that Saccharomyces cerevisiae is the only species responsible for alcoholic fermentation of fruit juices (13). In addition, this species has long been the organism of choice for use as a model of the eukaryotic cell in biochemistry, physiology, genetics, and molecular biology (24).

Genetic analysis has shown that the variable phenotypic expression of performance characteristics (including fermentation and assimilation) often observed in Saccharomyces strains may result from the presence of multiple gene loci that can be active, silent, or missing in different strains of the same species $(6,8,10,17,19)$. This may explain why definite identification of an unknown isolate as $S$. cerevisiae by conventional taxonomy is often difficult. In addition, these yeasts, which have been domesticated as a result of millennia of exploitation in human-created, industrial environments, are often homothallic or aneuploid and therefore are very difficult to use as models for genetic study and strain improvement.

Over the years the variability in the physiological performance of yeasts has resulted in a fluctuating classification. The 20 different species accepted at the beginning of this century were in 1970 reduced to 8 species by van der Walt (30), who described 13 new taxa and coined the term Saccharomyces sensu stricto. In 1984 Yarrow (38) combined the 21 species described by van der Walt in 1970 into one species because he found that the organisms were ecologically, physiologically, and technologically identical.

A series of subsequent nuclear DNA (nDNA)-nDNA reassociation studies revealed that the 21 previously described taxa belonging to the sensu stricto group, which made up the species $S$. cerevisiae sensu Yarrow (38), included at least the following four variably related species: Saccharomyces bayanus, S. cerevisiae, Saccharomyces paradoxus, and Saccharomyces

\footnotetext{
* Corresponding author. Mailing address: Dipartimento di Biologia Vegetale, University of Perugia, Borgo 20 Giugno 74, I-06121 Perugia, Italy. Phone: 39 (75) 585-6483. Fax: 39 (75) 585-6470.

$\dagger$ Paper no. 1771 of the Italian National Council for Research Special Project RAISA, Subproject 4.
}

pastorianus $(25,31-35)$. The genomes of some of these organisms exhibit intermediate levels of nucleotide sequence homology, as follows: $S$. bayanus and $S$. pastorianus, $72 \%$; and $S$. cerevisiae and $S$. pastorianus or $S$. paradoxus about $50 \%$. The levels of nDNA relatedness between $S$. bayanus and $S$. cerevisiae and between $S$. paradoxus and $S$. bayanus or $S$. pastorianus are consistently very low. These findings were partially confirmed by the results of chromosomal and gene locus polymorphism studies $(7,8,21,22)$.

In contrast to the three other members of Saccharomyces sensu stricto, which are associated only with industrial fermentation plants (13), $S$. paradoxus is a naturally occurring species which has been isolated from environments such as birch sap, oak exudates, soil, and Drosophila sp. Other salient differences between $S$. paradoxus and the other Saccharomyces species are the ability of $S$. paradoxus to sporulate abundantly in any type of medium and the $S$. paradoxus cell size (the cells are generally smaller than the cells of domesticated species) (31).

The presence of at least four taxa with different genomes within the thousands of $S$. cerevisiae strains conserved in culture collections and research laboratories throughout the world may explain the high levels of intraspecific polymorphism always encountered in electrophoretic karyotype studies performed with industrial and collection strains $(1,9,11,27)$.

In order to eliminate any possible cause of error resulting from misidentification of cultures concerning the polymorphism of karyotypes found within $S$. cerevisiae, we performed a comparative electrophoretic karyotyping study with eight certified strains (i.e., strains identified by nDNA-nDNA reassociation) of each of the four species ( $S$. bayanus, $S$. cerevisiae, $S$. paradoxus, $S$. pastorianus) of the sensu stricto group of the genus Saccharomyces.

\section{MATERIALS AND METHODS}

Microorganisms. All yeast cultures were obtained from the Industrial Yeasts Collection DBVPG, which is affiliated with the Dipartimento di Biologia Vegetale, University of Perugia, Perugia, Italy. Organisms were assigned to the four sensu stricto species on the basis of the results of previous nDNAnDNA reassociation studies (34). In this study we used the following strains (the values in parentheses are the levels of DNA relatedness to the respective type strains; the corresponding Centraalbureau voor Schimmel-cultures designations 


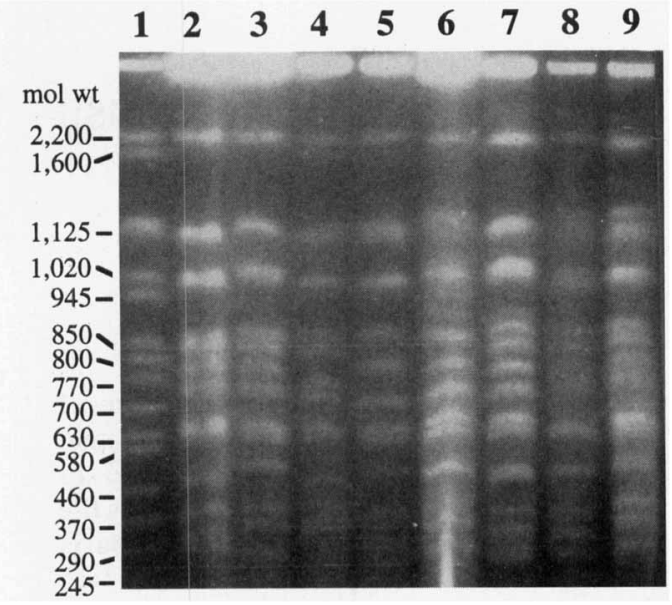

FIG. 1. CHEF electrophoresis of eight certified strains of $S$. cerevisiae. Lane 1, molecular weight marker (S. cerevisiae YNN 295); lane 2, DBVPG 6039; lane 3, DBVPG 6042; lane 4, DBVPG 6046; lane 5, DBVPG 6173 ${ }^{\mathrm{T}}$; lane 6, DBVPG 6220; lane 7, DBVPG 6249; lane 8, DBVPG 6251; lane 9, DBVPG 6254. The following conditions were used: gradient, $6 \mathrm{~V} / \mathrm{crn}$; temperature, $15^{\circ} \mathrm{C}$; time, $28 \mathrm{~h}$; switch, $16 \mathrm{~h}, 60$ $\mathrm{s}, 12 \mathrm{~h}, 90 \mathrm{~s}$

have been reported previously [31, 34]): $S$. cerevisiae Meyen ex Hansen DBVPG $6173^{\mathrm{T}}$ ( $\mathrm{T}=$ type strain) $(100 \%)$, DBVPG 6039 (90\%), DBVPG 6042 (87\%), DBVPG $6046(96 \%)$, DBVPG 6220 (93\%), DBVPG 6249 (96\%), DBVPG 6251 (89\%), and DBVPG 6254 (87\%); S. bayanus Saccardo DB VPG $6171^{\mathrm{T}}(100 \%)$, DBVPG $6730(93 \%)$, DBVPG 6179 (98\%), DBVPG 6131 (94\%), DBVPG 6260 (94\%), DBVPG $6253(94 \%)$, DBVPG $6259(90 \%)$, and DBVPG $6348(89 \%) ; S$. pastorianus Reess ex Hansen DBVPG $6047^{\mathrm{T}}(100 \%)$, DBVPG 6282 (85\%), DBVPG 6283 (79\%), DBVPG 6284 (98\%), DBVPG 6285 (78\%), DBVPG 6257 (99\%), DBVPG 6258 $(100 \%)$, and DBVPG $6251(87 \%)$; and S. paradoxus Batschinskaya DBVPG 6045 ${ }^{\mathrm{T}}$ (100\%), DBVPG 6304 (79\%), DBVPG $6466(75 \%)$, DBVPG 6489 (95\%), DBVPG $6490(93 \%)$, DBVPG 6491 (54\%), DBVPG 6493 (60\%), and DBVPG 6494 $(40 \%)$.

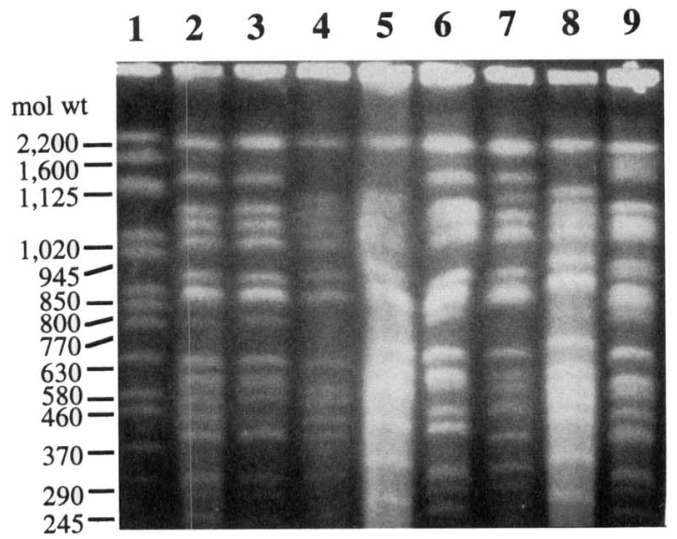

FIG. 2. CHEF electrophoresis of eight certified strains of $S$. bayanus. Lane 1, molecular weight marker ( S. cerevisiae YNN 295); lane 2, DBVPG 6171 ; lane 3, DBVPG 6730; lane 4, DBVPG 6179; lane 5, DBVPG 6131; lane 6, DBVPG 6260; lane 7, DBVPG 6253; lane 8, DBVPG 6259; lane 9, DBVPG 6348. See the legend to Fig. 1 for the running conditions used.

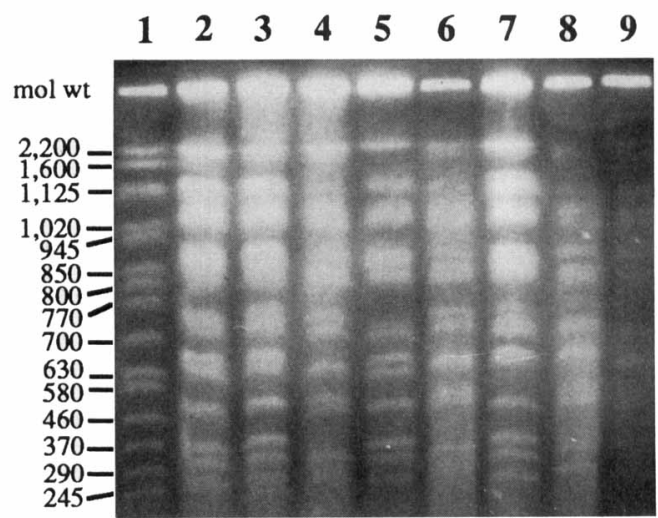

FIG. 3. CHEF electrophoresis of eight certified strains of $S$. pastorianus. Lane 1, molecular weight marker (S. cerevisiae YNN 295); lane 2, DBVPG 6282; lane 3, DBVPG 6283; lane 4, DBVPG 6284; lane 5, DBVPG 6285; lane 6, DBVPG 6047 ; lane 7, DBVPG 6257; lane 8, DBVPG 6258; lane 9, DBVPG 6261. See the legend to Fig. 1 for the running conditions used.

Preparation of chromosomal DNA for PFGE. To prepare chromosomal DNA for pulsed-field gel electrophoresis (PFGE), cells were grown overnight in $500-\mathrm{ml}$ bottles containing $50 \mathrm{ml}$ of yeast extract-peptone-dextrose medium on a rotary shaker $(150 \mathrm{rpm})$ at $25^{\circ} \mathrm{C}$. A total of $2 \times 10^{9}$ cells were harvested with a refrigerated centrifuge and washed sequentially with cold distilled, deionized water, and $5 \mathrm{ml}$ of cold $50 \mathrm{mM}$ EDTA. Then we used part of a new extraction procedure developed by workers in our laboratory (in press). Pellets were gently resuspended in 0.8 to $0.9 \mathrm{ml}$ of SPG $(50 \%$ glycerol in $10 \mathrm{mM}$ $\mathrm{NaH}_{2} \mathrm{PO}_{4}$ ) containing 12 to $25 \mathrm{mg}$ of lysing enzyme from Rhizoctonia solani (Sigma Chemical Co., St. Louis, Mo.) per $\mathrm{ml}$, and the preparations were incubated at $40^{\circ} \mathrm{C}$ in a water bath for $60 \mathrm{~min}$. Cell wall lysis $(>60 \%)$ was determined microscopically.

After lysis, each suspension was kept at $37^{\circ} \mathrm{C}$ for $5 \mathrm{~min}$ and then carefully mixed with $1 \mathrm{ml}$ of molten $1.8 \%$ LMP agarose (Sigma Chemical Co.) in $125 \mathrm{mM}$ EDTA before being pipetted into a $1.2-\mathrm{mm}$-thick plug mold. After solidification at 0 to $4^{\circ} \mathrm{C}$ for $20 \mathrm{~min}$, plugs were incubated in $2 \mathrm{ml}$ of LET $(500 \mathrm{mM}$

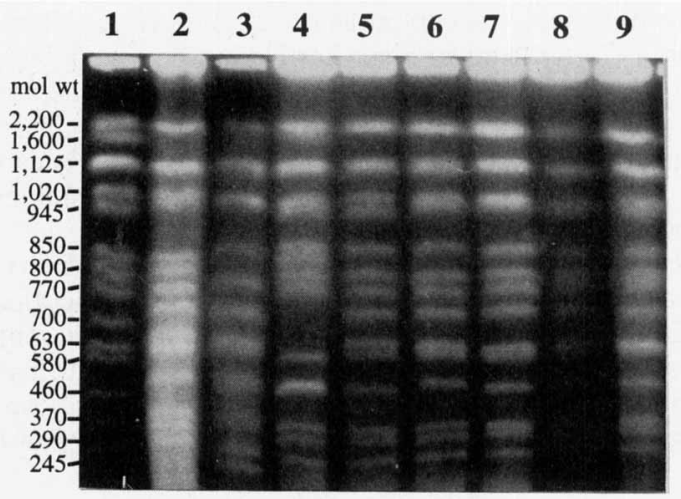

FIG. 4. CHEF electrophoresis of eight certified strains of $S$. paradoxus. Lane 1, molecular weight marker (S. cerevisiae YNN 295); lane 2, DBVPG 6304; lane 3, DBVPG 6045 ; lane 4, DBVPG 6466; lane 5, DBVPG 6489; lane 6, DBVPG 6490; lane 7, DBVPG 6491; lane 8, DBVPG 6493; lane 9, DBVPG 6494. See the legend to Fig. 1 for the running conditions used. 
TABLE 1. Electrophoretic bands of Saccharomyces sensu stricto strains clustered in molecular weight classes ${ }^{a}$

\begin{tabular}{|c|c|c|c|c|c|c|c|c|c|c|c|c|c|c|c|c|c|c|c|c|c|c|c|c|c|c|c|c|c|c|}
\hline \multicolumn{3}{|c|}{ Strain } & \multicolumn{28}{|c|}{ Presence of bands in the following classes: } \\
\hline Species & $\begin{array}{l}\text { Reference } \\
\text { no. }\end{array}$ & Designation & $\begin{array}{l}\frac{8}{0} \\
8 \\
0 \\
= \\
=\end{array}$ & & & & & $\begin{array}{l}\frac{5}{8} \\
8 \\
-1\end{array}$ & & $\frac{\frac{0}{3}}{\frac{8}{8}}$ & 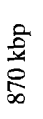 & $\begin{array}{l}\frac{\tilde{s}}{\vec{s}} \\
\frac{1}{\infty}\end{array}$ & $\begin{array}{l}\frac{0}{8} \\
\frac{8}{8}\end{array}$ & $\begin{array}{l}\frac{0}{8} \\
\stackrel{8}{2} \\
\stackrel{8}{8}\end{array}$ & 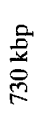 & $\begin{array}{l}\frac{2}{8} \\
\frac{8}{8}\end{array}$ & $\frac{\text { مे }}{\frac{8}{8}}$ & $\begin{array}{l}\text { 害 } \\
\stackrel{8}{8}\end{array}$ & $\begin{array}{l}\text { 商 } \\
\text { 总 }\end{array}$ & $\begin{array}{l}\frac{0}{8} \\
\frac{8}{8}\end{array}$ & $\begin{array}{l}\frac{8}{8} \\
\frac{8}{8} \\
i n\end{array}$ & & 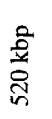 & $\begin{array}{l}\frac{0}{8} \\
\frac{8}{8} \\
\text { है }\end{array}$ & & 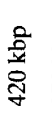 & $\frac{\text { مे }}{\frac{0}{2}}$ & & & $\frac{\frac{2}{2}}{\frac{2}{4}}$ \\
\hline \multirow[t]{8}{*}{ S. cerevisiae } & 1 & DBVPG 6039 & 0 & 1 & 0 & 0 & 0 & 1 & 0 & 0 & 1 & 0 & 0 & 1 & 0 & 1 & 0 & 0 & 1 & 1 & 0 & 0 & 0 & 1 & 0 & 1 & 1 & 1 & 0 & 0 \\
\hline & 2 & DBVPG 6042 & 0 & 1 & 0 & 0 & 0 & 1 & 0 & 0 & 1 & 0 & 0 & 1 & 0 & 1 & 0 & 0 & 1 & 0 & 0 & 0 & 0 & 1 & 0 & 1 & 1 & 1 & 0 & 0 \\
\hline & 3 & DBVPG 6046 & 0 & 1 & 0 & 0 & 0 & 1 & 0 & 0 & 1 & 0 & 1 & 1 & 0 & 1 & 0 & 0 & 0 & 1 & 0 & 0 & 0 & 1 & 0 & 1 & 1 & 1 & 0 & 0 \\
\hline & 4 & DBVPG $6173^{\mathrm{T}}$ & 0 & 1 & 0 & 0 & 0 & 1 & 0 & 0 & 1 & 0 & 1 & 0 & 1 & 1 & 0 & 0 & 0 & 1 & 0 & 0 & 0 & 1 & 0 & 0 & 0 & 1 & 1 & 1 \\
\hline & 5 & DBVPG 6220 & 0 & 1 & 0 & 0 & 0 & 1 & 0 & 0 & 1 & 0 & 0 & 1 & 0 & 1 & 0 & 0 & 0 & 1 & 1 & 0 & 0 & 1 & 0 & 1 & 1 & 1 & 1 & 0 \\
\hline & 6 & DBVPG 6249 & 0 & 1 & 0 & 0 & 0 & 1 & 1 & 1 & 1 & 0 & 0 & 1 & 0 & 1 & 0 & 0 & 1 & 1 & 0 & 0 & 0 & 1 & 0 & 1 & 0 & 1 & 0 & 0 \\
\hline & 7 & DBVPG 6251 & 0 & 1 & 0 & 0 & 0 & 1 & 0 & 0 & 1 & 1 & 0 & 1 & 1 & 0 & 0 & 0 & 0 & 1 & 0 & 0 & 0 & 1 & 0 & 1 & 1 & 1 & 0 & 0 \\
\hline & 8 & DBVPG 6254 & 1 & 1 & 0 & 0 & 0 & 1 & 0 & 0 & 1 & 1 & 0 & 1 & 1 & 0 & 0 & 0 & 1 & 1 & 0 & 0 & 0 & 1 & 0 & 1 & 1 & 1 & 0 & 0 \\
\hline \multirow[t]{8}{*}{ S. bayanus } & 9 & DBVPG $6171^{\mathrm{T}}$ & 1 & 0 & 0 & 1 & 1 & 1 & 0 & 1 & 1 & 0 & 1 & 0 & 0 & 1 & 1 & 0 & 1 & 0 & 1 & 0 & 0 & 0 & 1 & 0 & 1 & 1 & 1 & 0 \\
\hline & 10 & DBVPG 6730 & 1 & 0 & 0 & 1 & 1 & 1 & 0 & 1 & 1 & 0 & 1 & 0 & 0 & 1 & 1 & 0 & 1 & 0 & 1 & 0 & 0 & 0 & 1 & 0 & 1 & 1 & 1 & 0 \\
\hline & 11 & DBVPG 6179 & 0 & 0 & 1 & 1 & 1 & 1 & 0 & 1 & 1 & 0 & 0 & 0 & 0 & 1 & 0 & 1 & 1 & 0 & 1 & 0 & 0 & 0 & 1 & 0 & 1 & 0 & 1 & 0 \\
\hline & 12 & DBVPG 6131 & 0 & 0 & 1 & 1 & 1 & 1 & 0 & 1 & 1 & 0 & 0 & 0 & 1 & 1 & 0 & 0 & 1 & 0 & 0 & 1 & 1 & 0 & 0 & 1 & 1 & 0 & 0 & 0 \\
\hline & 13 & DBVPG 6260 & 1 & 0 & 1 & 1 & 1 & 0 & 0 & 1 & 1 & 0 & 0 & 0 & 0 & 1 & 1 & 0 & 0 & 0 & 1 & 0 & 0 & 1 & 0 & 1 & 1 & 1 & 0 & 0 \\
\hline & 14 & DBVPG 6253 & 1 & 1 & 0 & 1 & 1 & 0 & 1 & 0 & 0 & 1 & 0 & 0 & 0 & 1 & 1 & 0 & 1 & 1 & 0 & 0 & 0 & 0 & 1 & 0 & 1 & 1 & 1 & 0 \\
\hline & 15 & DBVPG 6259 & 0 & 1 & 1 & 1 & 1 & 0 & 1 & 1 & 0 & 0 & 1 & 0 & 1 & 0 & 1 & 1 & 1 & 0 & 1 & 0 & 0 & 0 & 1 & 0 & 1 & 0 & 1 & 0 \\
\hline & 16 & DBVPG 6348 & 1 & 0 & 1 & 1 & 1 & 0 & 1 & 0 & 1 & 0 & 1 & 0 & 0 & 1 & 1 & 1 & 0 & 1 & 0 & 0 & 1 & 0 & 0 & 1 & 1 & 0 & 0 & 0 \\
\hline \multirow[t]{8}{*}{ S. pastorianus } & 17 & DBVPG 6282 & 0 & 1 & 0 & 0 & 0 & 1 & 0 & 1 & 0 & 1 & 0 & 0 & 1 & 1 & 0 & 1 & 1 & 0 & 0 & 0 & 0 & 0 & 1 & 0 & 1 & 0 & 1 & 1 \\
\hline & 18 & DBVPG 6283 & 0 & 1 & 0 & 0 & 0 & 1 & 0 & 1 & 0 & 1 & 0 & 0 & 1 & 1 & 0 & 1 & 1 & 0 & 0 & 0 & 0 & 0 & 1 & 0 & 0 & 1 & 1 & 0 \\
\hline & 19 & DBVPG 6284 & 0 & 1 & 0 & 0 & 0 & 1 & 0 & 1 & 0 & 1 & 0 & 0 & 1 & 1 & 0 & 0 & 1 & 0 & 0 & 0 & 0 & 1 & 1 & 0 & 1 & 1 & 0 & 0 \\
\hline & 20 & DBVPG 6285 & 0 & 1 & 0 & 0 & 0 & 1 & 0 & 1 & 0 & 1 & 0 & 0 & 1 & 1 & 0 & 1 & 1 & 0 & 0 & 0 & 0 & 0 & 1 & 0 & 1 & 1 & 1 & 0 \\
\hline & 21 & DBVPG $6047^{\mathrm{T}}$ & 0 & 1 & 0 & 0 & 0 & 1 & 0 & 1 & 0 & 1 & 0 & 0 & 1 & 1 & 0 & 0 & 1 & 0 & 0 & 0 & 0 & 1 & 1 & 0 & 0 & 1 & 1 & 0 \\
\hline & 22 & DBVPG 6257 & 0 & 1 & 1 & 0 & 0 & 1 & 0 & 0 & 1 & 1 & 0 & 0 & 0 & 1 & 0 & 1 & 0 & 0 & 0 & 0 & 0 & 0 & 1 & 0 & 1 & 1 & 1 & 0 \\
\hline & 23 & DBVPG 6258 & 0 & 0 & 0 & 1 & 1 & 1 & 0 & 1 & 0 & 1 & 0 & 1 & 1 & 0 & 1 & 0 & 0 & 0 & 0 & 0 & 0 & 1 & 0 & 0 & 1 & 1 & 0 & 0 \\
\hline & 24 & DBVPG 6261 & 0 & 0 & 0 & 1 & 1 & 1 & 0 & 1 & 0 & 1 & 0 & 0 & 0 & 0 & 1 & 1 & 1 & 0 & 0 & 1 & 0 & 1 & 0 & 0 & 1 & 1 & 0 & 0 \\
\hline \multirow[t]{8}{*}{ S. paradoxus } & 25 & DBVPG 6304 & 0 & 1 & 0 & 0 & 1 & 1 & 0 & 0 & 1 & 0 & 1 & 1 & 1 & 1 & 0 & 0 & 1 & 0 & 0 & 0 & 1 & 0 & 1 & 0 & 1 & 1 & 0 & 0 \\
\hline & 26 & DBVPG $6045^{\mathrm{T}}$ & 0 & 1 & 0 & 0 & 1 & 1 & 0 & 1 & 0 & 0 & 1 & 0 & 1 & 0 & 1 & 0 & 0 & 0 & 0 & 0 & 1 & 0 & 0 & 1 & 0 & 1 & 1 & 0 \\
\hline & 27 & DBVPG 6466 & 0 & 1 & 0 & 0 & 1 & 1 & 0 & 1 & 1 & 0 & 1 & 1 & 0 & 0 & 0 & 0 & 1 & 0 & 0 & 0 & 0 & 1 & 0 & 0 & 1 & 1 & 1 & 0 \\
\hline & 28 & DBVPG 6489 & 0 & 1 & 0 & 0 & 1 & 1 & 0 & 1 & 0 & 1 & 1 & 0 & 1 & 1 & 1 & 0 & 0 & 0 & 0 & 0 & 1 & 0 & 0 & 1 & 1 & 0 & 1 & 0 \\
\hline & 29 & DBVPG 6490 & 0 & 1 & 0 & 0 & 1 & 1 & 0 & 1 & 0 & 1 & 1 & 0 & 1 & 0 & 1 & 0 & 0 & 0 & 0 & 0 & 1 & 0 & 0 & 1 & 1 & 0 & 1 & 0 \\
\hline & 30 & DBVPG 6491 & 0 & 1 & 0 & 0 & 1 & 1 & 0 & 1 & 0 & 1 & 1 & 0 & 1 & 0 & 1 & 0 & 0 & 0 & 0 & 0 & 1 & 0 & 0 & 1 & 1 & 1 & 0 & 0 \\
\hline & 31 & DBVPG 6493 & 0 & 1 & 0 & 0 & 1 & 1 & 0 & 1 & 0 & 1 & 1 & 0 & 1 & 0 & 1 & 0 & 0 & 0 & 0 & 0 & 1 & 0 & 0 & 1 & 0 & 1 & 0 & 0 \\
\hline & 32 & DBVPG 6494 & 0 & 1 & 0 & 0 & 1 & 1 & 0 & 1 & 0 & 1 & 1 & 0 & 1 & 0 & 1 & 0 & 0 & 0 & 0 & 0 & 1 & 0 & 1 & 1 & 0 & 1 & 0 & 0 \\
\hline
\end{tabular}

${ }^{a} 0$, band not present; 1 , band present.

EDTA, $10 \mathrm{mM}$ Tris; $\mathrm{pH} 7.5$ ) at $30^{\circ} \mathrm{C}$ in a water bath for 2 to 4 $\mathrm{h}$ and then washed once in cold $50 \mathrm{mM}$ EDTA and incubated overnight in NDS (500 mM EDTA, $500 \mathrm{mM}$ Tris, $1 \%$ lauryl sarcosine) at $50^{\circ} \mathrm{C}$ in the presence of $1 \mathrm{mg}$ of proteinase $\mathrm{K}$ (Sigma Chemical Co.) per $\mathrm{ml}$. The following day the plugs were rinsed in cold $50 \mathrm{mM}$ EDTA and stored at $4^{\circ} \mathrm{C}$ in $500 \mathrm{mM}$ EDTA (pH 9).

PFGE. PFGE was performed with a contour-clamped homogeneous electric field (CHEF) apparatus (Bio-Rad model CHEF-DR II). The gels used consisted of $1 \%$ agarose in $0.5 \times$ TBE ( $89 \mathrm{mM}$ boric acid, $89 \mathrm{mM}$ Tris $\mathrm{HCl}, 2 \mathrm{mM}$ EDTA). The operating conditions used are described in the legend to Fig. 1. The gels were stained with ethidium bromide and photographed by using conventional procedures.

Calculation of molecular weights of bands. Calibration curves were calculated for each gel by performing a polynomial regression analysis, using the observed migration distances and the previously reported molecular weights of the bands obtained with Bio-Rad marker strain YNN 295. The molecular weights of all of the bands observed in the sensu stricto yeast culture gels were determined by using the actual migration distances and the regression curve.

PCA. For the principal-component analysis (PCA) the molecular weights of all of the bands observed in the 32 sensu stricto strains used were grouped into 30 classes representing all of the molecular weights found in the certified sensu stricto strains. However, the 2,200- and 1,600-kbp classes were omitted from the matrix for the reasons discussed below. The data were placed in a binary matrix as either 1 (band present in the class) or 0 (band absent). The four clusters established on the basis of the certified model ( 32 sensu stricto strains) were used as references to assess the localization of profiles obtained from unknown, noncertified yeasts. A PCA was carried out by using the statistical package SIMCA-4 PCA (37) to define discrete clusters of strains.

\section{RESULTS AND DISCUSSION}

The electrophoretic karyotypes of the 32 strains used in this study are shown in Fig. 1 through 4; the karyotypes are shown separately for each of the four species assigned to the sensu stricto group by nDNA-nDNA reassociation (34).

All of the karyotypes contained high numbers of chromosomal bands, with molecular sizes ranging from approximately 250 to 2,200 kbp. Specifically, the electrophoretic profiles of $S$. cerevisiae strains contained 12 to 14 bands, the profiles of $S$. bayanus strains contained 14 to 17 bands, the profiles of $S$. 
pastorianus strains contained 11 to 13 bands, and the profiles of $S$. paradoxus strains contained 12 to 14 bands.

Figures 1 through 4 show that none of the species could be distinguished by the consistent presence or absence of a unique band or cluster of bands. Some examples of bands that species had in common are the $1,160-\mathrm{kbp}$ band that was present in five strains of $S$. bayanus and in one strain of $S$. cerevisiae and the $1,070-\mathrm{kbp}$ band that was found in all $S$. bayanus strains and two strains of $S$. pastorianus. This finding seems to eliminate the possibility that these species could be distinguished on the basis of the numbers of chromosomal bands and the molecular weight ranges of bands, even though each of the $S$. bayanus strains produced a slightly higher number of bands than the other strains examined. In any case, various authors have previously examined $S$. cerevisiae strains identified only by conventional taxonomic techniques and found several bands at molecular sizes less than $500 \mathrm{kbp}$, bands which were observed in all of the strains included in our model defined by molecular taxonomic techniques. This property clearly separates the four taxa of Saccharomyces sensu stricto from all of the nonSaccharomyces yeast species that have been examined so far by PFGE, all of which contain only a few, large chromosomal units $(2,7,9,14,15,26,27,35)$.

The results of genetic analyses of a few strains belonging to the sensu stricto group (20-22) also suggested that there are distinct species. Additional studies, however, revealed that variations also occur in the organization and arrangement of chromosomal bands in the $S$. cerevisiae electrophoretic genome $(8,36)$. Strain-to-strain polymorphism in electrophoretic profiles was observed both in the form of slightly different migration values for corresponding chromosomal bands and in the form of appearance or loss of single electrophoretic units. Even though the patterns obtained for strains belonging to a given species were polymorphic for number and molecular weights of the separated units, the inclusion in each run of a reference strain (Bio-Rad strain YNN 295) resulted in an acceptable level of reproducibility in the band molecular weight calculations. Polymorphic patterns can be used as data for multivariate analysis for discrete separation of strains into clusters.

The calculated data were grouped into 30 molecular weight classes of values that were actually observed in at least one strain. The classes corresponding to chromosome XII $(2,200$ $\mathrm{kbp})$ and chromosome IV (1,600 kbp) were excluded from further analysis for the following reasons. The 2,200-kbp band is consistently present in all of the yeasts that have been analyzed so far. In addition, since this band is localized in the nonlinear migration areas of gels, where differences in molecular weight are not easy to calculate, it does not display polymorphism. Chromosomes IV $(1,600 \mathrm{kbp})$ was clearly discernible as a singlet in only one strain of $S$. bayanus (Fig. 2, lane 9) and one strain of $S$. cerevisiae (Fig. 1, lane 4) and as a doublet in only one strain of $S$. paradoxus (Fig. 4, lane 4). This chromosomal unit has been reported to exhibit migration anomalies (28) due to trapping and band inversion. Furthermore, it seems to be indispensable according to the latest genetic map of $S$. cerevisiae (18) that assigns to chromosome IV single copies of several cell division cycle-related genes. As a consequence of the considerations described above, the 1,600 $\mathrm{kbp}$ band was considered to be always present, although it could not be separated as a singlet and therefore was excluded from the statistical analysis.

The data for the PCA were organized in a binomial matrix in which the chromosomal bands observed in the 32 strains belonging to the sensu stricto group of the genus Saccharomyces were distributed in 28 molecular weight classes. The matrix

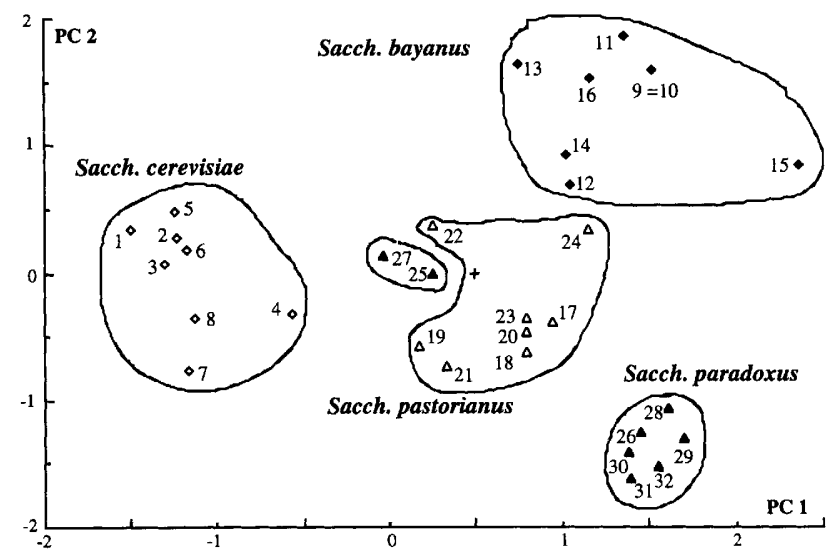

FIG. 5. Distribution of the sensu stricto strains in a statistical two-dimensional space as determined by a PCA. PC, principal component.

is shown integrally in Table 1 for the sake of further PCA and identification by fitting unknown strains into one of the clusters defined by this working model.

The plot shown in Fig. 5 was obtained by using the two principal components identified by statistical analysis. The distribution of the 32 strains into the four separate clusters that were resolved on the plot is in a general agreement with the classification of these organisms into the four sensu stricto taxa based on nDNA data. The only apparent exceptions were $S$. paradoxus DBVPG 6466 and DBVPG 6304, whose coordinates fall in the vicinity of $S$. pastorianus; these two yeasts, however, exhibit DNA relatedness values with the type strain of $S$. paradoxus strain DBVPG 6045, of 79 and $75 \%$, respectively, values that are very close to the approximate conspecificity level of $80 \%$ commonly accepted in yeast classification (23). The levels of genomic relatedness between $S$. paradoxus DB VPG 6466 and DBVPG 6304 and other Saccharomyces species are as follows: $S$. cerevisiae, 59 and $49 \%$, respectively; $S$. bayanus, 26 and 33\%, respectively; and $S$. pastorianus, 8 and $19 \%$, respectively (31). A simple, visual examination of the karyotypes of DBVPG 6466 and DBVPG 6304 (Fig. 4, lanes 4 and 8 ), as well as the distribution of molecular weight classes (Table 1), revealed a genome organization different from that exhibited by the six other certified $S$. paradoxus strains.

An analogous situation was recently encountered in our laboratory in a study of other species of the genus Saccharomyces. Three strains of Saccharomyces exiguus that exhibited 68 to $76 \%$ DNA relatedness were found to have slightly different karyotypes (35), while several strains of Saccharomyces servazzii that were conspecific with the type strain of the species produced different PFGE profiles (unpublished data). Additional studies will be necessary to resolve these anomalous correlations between levels of nDNA relatedness and genome organization.

For the time being, we propose that the results of a fitting analysis of the electrokaryotype may be used to assign each unknown yeast strain to one of the four sensu stricto subgroups and that this preliminary information should be integrated with the results of a few conventional taxonomic tests. In fact, the results of a recent study of the discriminating power of conventional physiological tests for the certified organisms used in this study (34) showed that $S$. bayanus is the only species which can grow in a vitamin-free medium. Maximum growth temperatures separate $S$. bayanus and $S$. pastorianus, 
TABLE 2. Results of a fitting analysis of the sensu stricto strains as determined by $\mathrm{PCA}^{a}$

\begin{tabular}{|c|c|c|c|c|c|c|}
\hline \multicolumn{3}{|c|}{ Strain } & \multicolumn{4}{|c|}{ RSD value with: } \\
\hline Species & Reference no. & Designation & S. cerevisiae & S. bayanus & S. pastorianus & S. paradoxus \\
\hline \multirow[t]{8}{*}{ S. cerevisiae } & 1 & DBVPG 6039 & 0.1139 & 0.5961 & 0.5427 & 0.7075 \\
\hline & 2 & DBVPG 6042 & 0.1802 & 0.5820 & 0.5061 & 0.6798 \\
\hline & 3 & DBVPG 6046 & 0.2121 & 0.6031 & 0.5788 & 0.6509 \\
\hline & 4 & DBVPG $6173^{\mathrm{T}}$ & 0.1087 & 0.6541 & 0.5576 & 0.6170 \\
\hline & 5 & DBVPG 6220 & 0.2645 & 0.6081 & 0.5736 & 0.6872 \\
\hline & 6 & DBVPG 6249 & 0.2542 & 0.6218 & 0.5736 & 0.6962 \\
\hline & 7 & DBVPG 6251 & 0.1384 & 0.6584 & 0.5123 & 0.6005 \\
\hline & 8 & DBVPG 6254 & 0.1287 & 0.6465 & 0.5396 & 0.6615 \\
\hline \multirow[t]{8}{*}{ S. bayanus } & 9 & DBVPG $6171^{\mathbf{T}}$ & 0.6677 & 0.1949 & 0.5505 & 0.6615 \\
\hline & 10 & DBVPG 6730 & 0.6677 & 0.1949 & 0.5505 & 0.6615 \\
\hline & 11 & DBVPG 6179 & 0.6879 & 0.2707 & 0.4726 & 0.7369 \\
\hline & 12 & DBVPG 6131 & 0.6582 & 0.2601 & 0.5755 & 0.6186 \\
\hline & 13 & DBVPG 6260 & 0.6330 & 0.3756 & 0.5553 & 0.7075 \\
\hline & 14 & DBVPG 6253 & 0.6725 & 0.2482 & 0.5470 & 0.7026 \\
\hline & 15 & DBVPG 6259 & 0.7969 & 0.4334 & 0.5975 & 0.6464 \\
\hline & 16 & DBVPG 6348 & 0.7174 & 0.2227 & 0.6457 & 0.7057 \\
\hline \multirow[t]{8}{*}{ S. pastorianus } & 17 & DBVPG 6282 & 0.6241 & 0.5793 & 0.2567 & 0.6004 \\
\hline & 18 & DBVPG 6283 & 0.5913 & 0.5694 & 0.1667 & 0.5873 \\
\hline & 19 & DBVPG 6284 & 0.4976 & 0.5778 & 0.2022 & 0.5684 \\
\hline & 20 & DBVPG 6285 & 0.5819 & 0.5345 & 0.1390 & 0.5830 \\
\hline & 21 & DBVPG $6047^{\mathrm{T}}$ & 0.5228 & 0.5855 & 0.1741 & 0.5873 \\
\hline & 22 & DBVPG 6257 & 0.5412 & 0.5514 & 0.1407 & 0.6747 \\
\hline & 23 & DBVPG 6258 & 0.5679 & 0.5687 & 0.2039 & 0.5327 \\
\hline & 24 & DBVPG 6261 & 0.6681 & 0.5338 & 0.2216 & 0.6318 \\
\hline \multirow[t]{8}{*}{ S. paradoxus } & 25 & DBVPG 6304 & 0.5190 & 0.5504 & 0.5335 & 0.5767 \\
\hline & 26 & DBVPG $6045^{\mathrm{T}}$ & 0.5902 & 0.5844 & 0.5566 & 0.0280 \\
\hline & 27 & DBVPG 6466 & 0.4577 & 0.5349 & 0.4912 & 0.5814 \\
\hline & 28 & DBVPG 6489 & 0.6195 & 0.5449 & 0.5222 & 0.0944 \\
\hline & 29 & DBVPG 6490 & 0.6215 & 0.5718 & 0.5279 & 0.0987 \\
\hline & 30 & DBVPG 6491 & 0.5833 & 0.5734 & 0.4827 & 0.0120 \\
\hline & 31 & DBVPG 6493 & 0.6007 & 0.6060 & 0.5099 & 0.0882 \\
\hline & 32 & DBVPG 6494 & 0.6319 & 0.6066 & 0.5099 & 0.0119 \\
\hline
\end{tabular}

a The RSD value for each strain was compared with the reference RSD values for the four species belonging to the sensu stricto group. The reference RSD values for $S$. cerevisiae, $S$. bayanus, $S$. pastorianus, and $S$. paradoxus were $0.3952,0.4585,0.3070$, and 0.1931 , respectively. A strain was assigned to one of the four species when its RSD value was equal to or less than the species reference RSD value.

which never grow at temperatures above $34^{\circ} \mathrm{C}$, from $S$. cerevisiae and $S$. paradoxus, which always grow at $37^{\circ} \mathrm{C}$. S. cerevisiae can be distinguished from $S$. paradoxus because the latter is able to grow on D-mannitol as a sole carbon source. Integration of results from all of the tests described above may provide more accurate and unequivocal assignments, especially for those organisms that exhibit one or more exceptions to the discriminating characteristics described above.

To verify analytically the coherence of strain assignments to clusters in view of the possible use of karyotyping for fast identification of previously unidentified sensu stricto cultures, a fitting analysis was carried out as a corollary to the PCA. Table 2 shows the fitting values which we calculated for each of the yeasts studied. On the basis of these data discrete separation of the strains into their respective taxa, as originally defined by nDNA reassociation, was obtained. The only exceptions were the two strains of $S$. paradoxus discussed above, DBVPG 6304 and DBVPG 6466, which had fitting values that indicated that they were more closely associated with the $S$. pastorianus universe.

All of the yeasts which we studied except $S$. paradoxus, which is apparently associated only with natural environments (19), have been isolated almost exclusively from surfaces in fermentation plants (13). Natural sugary juices are an exceedingly favorable substrate for members of this group of yeasts because of the highly selective action of a low $\mathrm{pH}(\mathrm{pH} 3.5)$, the high sugar concentration, and the resistance of the organisms to ethanol produced by fermentation. The long association with this carbon and energy source is a possible explanation for the loss of sporulating ability by industrial cultures.

The considerations described above challenge the commonly accepted biological species concept, which is based on reproductive isolation among populations (4). In our model of practically asexual yeasts that reproduce by fermentation only in confined man-made niches (13), it is likely that the main driving force of speciation is based on a somatogamous mechanism of some sort. The coexistence of chromosome sets that have different origins in the same genome has been determined previously for several sensu stricto strains by different genetic approaches $(21,32)$. In addition, chromosomal rearrangement during vegetative growth of a wild strain of $S$. cerevisiae has also been preliminarily demonstrated by PFGE (12).

If the evolutionary scheme described above has been in operation for at least the last 6,000 to 7,000 years, it is impossible to apply the commonly accepted biological species concept to these yeasts. In fact, these organisms should be regarded as the variable expressions of several rearrangements 
TABLE 3. RSD values for four test strains compared with the reference RSD values for the species belonging to the sensu stricto group of the genus Saccharomyces ${ }^{a}$

\begin{tabular}{lcccc}
\hline \multirow{2}{*}{ Strain } & \multicolumn{4}{c}{ RSD value with: } \\
\cline { 2 - 5 } & S. cerevisiae & S. bayanus & S. pastorianus & S. paradoxus \\
\hline Wine yeast strain I & 0.3969 & 0.6570 & 0.6095 & 0.6765 \\
Wine yeast strain II & 0.3785 & 0.6439 & 0.5029 & 0.7040 \\
DBVPG 6565 & 0.3620 & 0.6599 & 0.6177 & 0.6765 \\
DBVPG 6699 & 0.3730 & 0.6200 & 0.5503 & 0.7311 \\
\hline
\end{tabular}

${ }^{a}$ The reference RSD values for $S$. cerevisiae, $S$. bayanus, $S$. pastorianus, and $S$. paradoxus were $0.3952,0.4585,0.3070$, and 0.1931 , respectively.

of entire or fragmented chromosomes somatogamously sorted out during the billions and billions of generations that occur every year in wineries throughout the world. As a matter of fact, recent evidence has led several botanists to conclude that many plant species are asexual or apomictic and that more than $30 \%$ originated as hybrids (16).

In order to test the overall discriminating ability of our approach, karyotypes of additional cultures belonging to the species $S$. cerevisiae were added to the matrix in Table 1 . Two of these organisms were cultures that were recently isolated from local wineries where they are presently being tested for use as selected starters for winemaking. Conversely, DBVPG 6565 , which has been informally described as "Saccharomyces douglasii" nomen nudum, is a strain that is presently used in molecular genetics studies $(3,5)$, while DBVPG 6699, which has been informally described as "Saccharomyces boulardii" nomen nudum, is used as an antidiarrhea agent. Assignment of these organisms to one of the four species of our model was accomplished by computing the statistical distance (residual standard deviation [RSD] around the model class) of electrokaryotypes from the boundaries of each of the four universes discriminated in the model shown in Fig. 5. According to this analysis, an unknown strain belongs to a given species when its RSD value falls below the calculated reference RSD for the universe representing that species. The calculated statistical distances (Table 3) placed the yeast strains that were tested immediately outside the boundaries of the $S$. cerevisiae universe, but the strains were clearly separated from the remaining three universes.

Even though the uniqueness of the $S$. cerevisiae universe cannot be questioned on the basis of its compactness, the statistical model for comparison of $S$. cerevisiae karyotypes is far from being completely defined. In fact, the original cluster was obtained by using only a few collection cultures, which mainly represented type strains of some former species recently merged with the sensu stricto group of the genus Saccharomyces (33), while the actual number of $S$. cerevisiae strains conserved throughout the world may be estimated to be several thousand. As a consequence, we may expect a great deal of genomic polymorphism in industrial strains, which are apparently the result of accelerated evolution in segregated and unique environments (winery surfaces in geographically distinct areas) that provide exceptionally favorable growth conditions (fermenting grape musts) which are suitable only for an ethanol-tolerant microorganism such as $S$. cerevisiae.

\section{ACKNOWLEDGMENTS}

We thank Mario Bertuccioli, University of Basilicata, Potenza, Italy, for his valuable advice, as well as for the use of the statistical package SIMCA-4PA in his laboratory.

This research was supported by a grant from the Italian National Council for Research Special Project RAISA, Subproject 4.

\section{REFERENCES}

1. Bidenne, C., B. Blondin, S. Dequin, and F. Vezinhet. 1992. Analysis of the chromosomal DNA polymorphism of wine strains of Saccharomyces cerevisiae. Curr. Genet. 22:1-7.

2. De Jonge, P., F. C. M. De Jongh, R. Meijers, H. Y. Steensma, and W. A. Scheffers. 1986. Orthogonal-field-alternation gel electrophoresis banding patterns of DNA from yeasts. Yeast 2:193-204.

3. Delorme, M. O., A. Hénaut, and P. Vigier. 1988. Mutations in the NAM2 genes of Saccharomyces cerevisiae and S. douglasii are clustered nonrandomly as a result of constrains on the nucleic acid sequence and not on the protein. Mol. Gen. Genet. 213:310-314.

4. Dobzhansky, T. 1976. Organismic and molecular aspects of species formation, p. 95-105. In F. Ayala (ed.), Molecular evolution. Sinauer Associates, Sunderland, Mass.

5. Herbert, C. J., G. Dujardin, M. Labouesse, and P. P. Slonimski. 1988. Divergence of the mitochondrial leucyl tRNA synthase genes in two closely related yeasts, Saccharomyces cerevisiae and Saccharomyces douglasii: a paradigm of incipient evolution. Mol. Gen. Genet. 213:297-309.

6. Holmberg, S. 1982. Genetic differences between Saccharomyces carlsbergensis and Saccharomyces cerevisiae. Restriction endonuclease analysis of genes in chromosome III. Carlsberg Res. Commun. 47:233-244.

7. Johnston, J. R., C. R. Contopoulou, and R. K. Mortimer. 1988. Karyotyping of yeast strains of several genera by field inversion gel electrophoresis. Yeast 4:191-198.

8. Johnston, J. R., and R. K. Mortimer. 1986. Electrophoretic karyotyping of laboratory and commercial strains of Saccharomyces and other yeasts. Int. J. Syst. Bacteriol. 36:569-572.

9. Kaneko, Y., and I. Banno. 1991. Reexamination of Saccharomyces bayanus strains by DNA-DNA hybridization and electrophoretic karyotyping. Institute for Fermentation (Osaka) Res. Commun. 15:30-41.

10. Kaneko, Y., K. Mikata, and I. Banno. 1989. Karyotyping of Saccharomyces exiguus by pulsed-field electrophoresis. Institute for Fermentation (Osaka) Res. Commun. 14:111-117.

11. Kunze, G., I. Kunze, A. Barner, and R. Schulz. 1993. Classification of Saccharomyces cerevisiae strains by genetical and biochemical methods. Monatsschr. Brauwiss. 46:132-136.

12. Longo, E., and F. Vezineht. 1993. Chromosomal rearrangement during vegetative growth of a wild strain of Saccharomyces cerevisiae. Appl. Environ. Microbiol. 59:322-326.

13. Martini, A. 1993. Origin and domestication of the wine yeast Saccharomyces cerevisiae. J. Wine Res. 4:165-176.

14. Merz, W. G., C. Connelly, and P. Hieter. 1988. Variation of electrophoretic karyotypes among clinical isolates of Candida albicans. J. Clin. Microbiol. 26:842-845.

15. Miller, M., J. L. F. Kock, G. H. J. Pretorius, and D. J. Coetzee. 1989. The value of orthogonal-field-alternation gel electrophoresis and other criteria in the taxonomy of the genus Pichia Hansen emend. Kurtzman. Syst. Appl. Microbiol. 12:191-202.

16. Mitchell, R., D. Bleakly, R. Cabin, R. Chan, B. Enquist, D. Marshall, S. Reed, G. Stevens, and N. Waser. 1993. Species concepts. Nature (London) 364:20.

17. Monia, B. P., K. M. Haskell, J. R. Ecker, D. J. Ecker, and S. T. Crooke. 1989. Chromosomal mapping of the ubiquitin gene family in Saccharomyces cerevisiae by pulsed field gel electrophoresis. Nucleic Acids Res. 17:3611.

18. Mortimer, R. K., C. R. Contopoulou, and J. S. King. 1992. Genetic and physical maps of Saccharomyces cerevisiae, edition 11. Yeast 8:817-902.

19. Naumov, G. 1986. Genetic differentiation and ecology of the yeast Saccharomyces paradoxus. Dokl. Akad. Nauk SSSR 289:213216.

20. Naumov, G., E. S. Naumova, R. A. Lantto, E. J. Louis, and M. Korhola. 1992. Genetic homology between Saccharomyces cerevisiae and its sibling species $S$. paradoxus and $S$. bayanus: electrophoretic karyotypes. Yeast 8:599-612.

21. Nillson-Tillgren, T., C. Gjermansen, S. Holmberg, J. G. L. Petersen, and M. C. Kielland-Brandt. 1986. Analysis of chromosome $\mathrm{V}$ and the $I L V I$ gene from Saccharomyces carlsbergensis. Carlsberg Res. Commun. 51:309-326.

22. Pedersen, M. B. 1986. DNA sequence polymorphism in the genus 
Saccharomyces. IV. Homologous chromosome III of Saccharomyces bayanus, $S$. carlsbergensis, and $S$. uvarum. Carlsberg Res. Commun. 51:185-202.

23. Price, C. W., G. B. Fuson, and H. J. Phaff. 1978. Genome comparison in yeast systematics: delimitation of species within the genera Schwanniomyces, Saccharomyces, Debaryomyces, and Pichia. Microbiol. Rev. 42:161-193.

24. Roman, H. 1981. Development of yeast as an experimental organism, p. 1-9. In J. N. Strathern, E. W. Jones, and J. R. Broach (ed.), The molecular biology of the yeast Saccharomyces-1977. Cold Spring Harbor Laboratory, Cold Spring Harbor, N.Y.

25. Rosini, G., F. Federici, A. E. Vaughan, and A. Martini. 1982. Systematics of the species of the yeast genus Saccharomyces associated with the fermentation industry. Eur. J. Appl. Microbiol. Biotechnol. 15:188-193.

26. Rustchenko-Bulgac, E. P. 1991. Variations of Candida albicans electrophoretic karyotypes. J. Bacteriol. 173:6586-6596.

27. Sheehan, C. A., A. S. Weiss, I. A. Newson, V. Flint, and D. C. O'Donnel. 1991. Brewing yeast identification and chromosome analysis using high resolution chef gel electrophoresis. J. Inst. Brew. 97:163-167.

28. Slater, G. W., C. Turmel, M. Lalande, and J. Noolandi. 1989. DNA gel electrophoresis: effect of field intensity and agarose concentration on band inversion. Biopolymers 28:1793-1799.

29. Smith, C. L., and C. R. Cantor. 1987. Preparation and manipulation of large DNA molecules: advances and applications. Trends Biochem. Sci. 12:284-287.
30. van der Walt, J. P. 1970. Saccharomyces Meyen emend. Reess, p. 555-718. In J. Lodder (ed.), The yeasts. A taxonomic study. North-Holland Publishing Co., Amsterdam.

31. Vaughan Martini, A. 1989. Saccharomyces paradoxus comb. nov., a newly separated species of the Saccharomyces sensu stricto complex based upon nDNA/nDNA homologies. Syst. Appl. Microbiol. 12:179-182.

32. Vaughan Martini, A., and C. P. Kurtzman. 1985. Deoxyribonucleic acid relatedness among species of the genus Saccharomyces sensu stricto. Int. J. Syst. Bacteriol. 35:508-511.

33. Vaughan Martini, A., and A. Martini. 1987. Three newly delimited species of Saccharomyces sensu stricto. Antonie van Leeuwenhoek 53:77-84.

34. Vaughan Martini, A., and A. Martini. 1993. A taxonomic key for the genus Saccharomyces. Syst. Appl. Microbiol. 16:113-119.

35. Vaughan Martini, A., A. Martini, and G. Cardinali. 1993. Electrophoretic karyotyping as a taxonomic tool in the genus Saccharomyces. Antonie van Leeuwenhoek 63:145-156.

36. Vollrath, D., R. W. Davis, C. Connelly, and P. Hieter. 1988. Physical mapping of large DNA by chromosome fragmentation. Proc. Natl. Acad. Sci. USA 85:6027-6031.

37. Wold, S., and M. Sjostrom. 1977. SIMCA: a method for analysing chemical data in terms of similarity and analogy. ACS (Am. Chem. Soc.) Symp. Ser. 52:243-282.

38. Yarrow, D. 1984. Saccharomyces Meyen ex Reess, p. 379-395. In N. J. W. Kreger-van Rij (ed.), The yeasts: a taxonomic study, 3rd ed. Elsevier Science Publishers, Amsterdam. 